\title{
Ionic Channels in Biological Membranes: Natural Nanotubes Described by the Drift-Diffusion Equations
}

\author{
BOB EISENBERG* \\ Dept. of Molecular Biophysics and Physiology, Rush Medical Center, Chicago IL 60612
}

\begin{abstract}
An important class of biological molecules-proteins called ionic channels-conduct ions (like $\mathrm{Na}^{+}, \mathrm{K}^{+}, \mathrm{Cl}^{-}$) through a narrow tunnel of fixed charge ('doping'). Ionic channels are the main pathway by which substances move into cells and so are of great biological and medical importance: a substantial fraction of all drugs used by physicians act on channels. Channels can be studied in the tradition of computational electronics. Drift diffusion equations form an adequate model of IV relations of 6 different channel proteins in $\sim 10$ solutions over $\pm 150 \mathrm{mV}$. Ionic channels can also be studied with the powerful techniques of molecular biology. Atoms can be modified one at a time and the location of every atom can be determined. Ionic channels are natural nanotubes that can be controlled more precisely and easily than physical nanostructures but biologists need help if realistic simulations are to be done atomic detail.
\end{abstract}

Keywords: Biological membranes, ionic channels, drift diffusion equations, Gummel iteration

Drift-diffusion equations, combined with Poisson's equation $(P D D)$, are widely used in physical sciences to describe the flux of charge carriers through systems containing fixed charge (doping) [28]. An important class of biological moleculesproteins called ionic channels - conduct ions (like $\mathrm{Na}^{+}, \mathrm{K}^{+}, \mathrm{Cl}^{-}$), and thus current, through a narrow tunnel of fixed charge ('doping') formed by the polar residues of the protein [36] although electron flow plays no direct role in their conduction of current. These proteins can be studied with the full power of molecular biology [1]; for example, they can be modified one atom at a time with the techniques of molecular genetics. Thus, these natural nanotubes are a natural 'hole in the wall' that can be controlled more precisely and easily than many physical nanostructures.

Ionic channels open and close ('gate') to give currents that are a random telegraph signal [34]. The properties of gating are complex and the structure(s) and mechanism(s) that produce gating are not known [23], but the flow of ions through open channels is much simpler, and obeys the $P D D$ equations, as we shall see $[10-13,19,37]$.

Channels are the main pathway by which substances move in and out of cells and so are of

\footnotetext{
* Invited by and submitted to VLSI Design (Proceedings of the Fifth International Workshop on Computational Electronics).
} 
great biological and medical importance: they are responsible for signaling in the nervous system; for coordination of muscle contraction-including the coordination of cardiac muscle that allows the heart to function as a pump-and they are involved in transport in every cell and organ, for example, in the kidney, intestine and endocrine glands $[1,36]$. A substantial fraction of all drugs used by physicians act directly or indirectly on channels [35].

Channels are studied one molecule at a time in hundreds, if not thousands of laboratories every day [14-16, 31], using Neher \& Sakmann's patch clamp method $[6,32,34]$ (for which they received the Nobel Prize). The concentrations of ions outside channels (that carry current through the channel) can be directly controlled and the shape of current voltage (IV) relations can be manipulated. In this way, a wide range of IV behavior can be measured from the single doping profile of one type of channel and so much can be inferred about the doping profile from IV measurements (if they are taken in many different (pairs of) concentrations of current carriers). For all these reasons, channels are a popular object for experimentation: thousands (!) of abstracts describing their properties are presented each year at the annual meeting of the Biophysical Society and hundreds of papers are published about them, chiefly in the Biophysical Journal and the Journal of Physiology (London).

Channels are also an appealing and important object for theoretical analysis and numerical simulation. Open channels are probably the simplest protein structures of general biological importance. Unlike many other subjects of biophysical investigation, ionic channels are a general biological system with importance for every organ, tissue, and cell in an animal and plant. Indeed, they are probably just as important for subcellular organelles. Ionic channels are well defined biological systems that can be investigated both with the techniques of molecular biology and of biophysical chemistry, helped substantially by the techniques and insights of semiconductor physics, I believe.
Ionic movement plays an important role in the function of all proteins-e.g., enzymes-and so a model that describes ionic movement in channels is likely to give important insight into protein function in general. Indeed, the closely related Poisson-Boltzmann theory $[18,24]$ has been of considerable help already, even though it is a strictly equilibrium theory that does not permit flux at any time or location.

Theories of physical chemistry [4] and electrochemistry [30] certainly should be able to predict the movement of ions through a tunnel of fixed charge-a hole in the wall-on the biological time scale of $100 \mu \mathrm{sec}-10 \mathrm{sec}$, but they need to be supplemented by the theories and simulations of carrier transport in general, e.g., in semiconductors [28]. The physical chemical tradition has not often dealt with flux $[2,3,5,8,9,21,22,25,29]$ in such a system of fixed charge, whereas the flux of charge carriers has been the main subject of semiconductor physics and computational electronics for many years, if not decades.

Five laboratories have shown that the $P D D$ of semiconductor physics form an adequate model of IV relations of 6 different channel proteins in $\sim 10$ pairs of solutions (containing different concentrations of the charge carriers $\mathrm{Na}^{+}, \mathrm{K}^{+}, \mathrm{Cl}^{-}$) in the range $\pm 150 \mathrm{mV}[10-13,37]$. The IV relations are qualitatively different in different types of channels-some are linear, some sublinear and some superlinear-because different channel proteins have qualitatively different profiles of fixed charge arising from their different sequences of amino acids.

The structures of two of these proteins (porin and its mutant G-119D) are known from the standard methods of molecular biology: the location of every atom has been determined by X-ray diffraction $[17,26,27,33,38]$ with an accuracy of $\sim 0.1 \AA$. The mutant has one extra negative charge. Measurements of IV relations from a single molecule of porin [37] allow the $P D D$ model to estimate the additional charge as $-0.97 \mathrm{e}$, although this estimate will undoubtedly change as more work is done. (I hasten to add that no information 
about the proteins is used in the analysis except the length and diameter of the channel; parameters were not adjusted in any way.)

We conclude that the $P D D$ equations seem to be an adequate model open channels. It is surprising that the $P D D$ equations work as well as they do, given their evident inadequacies. I imagine they work this well because the fixed charge density of channels is large $\left(\sim 3 \times 10^{21} \mathrm{~cm}^{-3}\right)$ compared to the concentration of ions outside the channel $\left(2 \times 10^{19}\right.$ to $1 \times 10^{21} \mathrm{~cm}^{-3}$ ); because the biological range of voltages is quite limited $( \pm 200 \mathrm{mV})$; and because the $P D D$ model uses effective parameters. Eisenberg, Chen, and Schuss have recently shown how the $P D D$ equations can be derived in single file systems like channels that conduct one ion at a time. The $P D D$ equations, or equations quite like them, describe the mean properties of ensembles of Langevin equations, each of which specifies the motion of a single ion (of a particular type moving from a given side of the channel), each of which is coupled to its own reaction field described by a Poisson equation and boundary conditions.

The $P D D$ equations are just a first, low resolution description of open channels. More realistic models (using Monte Carlo simulations called molecular dynamics in the world of proteins $[7,8$, 20]) are needed to provide insight with atomic resolution. It is likely that many critical functions of channels and enzymes will be best understood this way-by atomic resolution simulations that include flux-but biologists cannot do the simulations themselves: their simulations of atomic resolution have been confined strictly to equilibrium as have those of most chemists. Much help is needed from the community of computational electronics if flux, and electrical potentials at the electrodes (i.e., boundaries) of the system, are to be included in chemical simulations of atomic detail.

Ionic channels are so important biologically, but so well defined physically, that they are an ideal object of biophysical investigation. The techniques of computational electronics and molecular biology can be joined together to determine how ionic channels work. Perhaps the same will prove true of many other chemical and biological systems, but it is wise to try the simple ones first. Nothing is likely to be simpler physically than a hole in the wall.

\section{Acknowledgment}

The adventure of the open channel has been shared Duan Chen every step of the way. I hope he has enjoyed our work together as much as I have. We are both grateful to Mark Lundstrom and Joe Jerome who introduced us to the community of computational electronics.

\section{References}

[1] Alberts, B. et al. (1994). Molecular Biology of the Cell Third ed. New York, Garland.

[2] Allen, M. P. and Tildesley, D. J. (1987). Computer Simulation of Liquids, New York, Oxford.

[3] Allen, M. P. and Tildesley, D. J. (1990). Computer Simulation in Chemical Physics, Series C: Mathematical and Physical Sciences, 397, Boston, Kluwer.

[4] Berry, S. R., Rice, S. A. and Ross, J. (1980). Physical Chemistry, New York, John Wiley \& Sons.

[5] Binder, K. (1995). The Monte Carlo Method in Condensed Matter Physics Second ed. Topics in Applied Physics, 71, New York, Springer.

[6] Boulton, A. A., Baker, G. B. and Walz, W. (1995). Patch Clamp Applications and Protocols, Totowa, NJ, Humana Press.

[7] Brooks, B. R. et al. (1983). CHARMM: a program for macromolecular energy minimization and dynamics calculations. J. Comput. Chem., 4, 187-217.

[8] Brooks, C. L., Karplus, M. and Pettitt, B. M. (1988). Proteins: A Theoretical Perspective of Dynamics, Structure and Thermodynamics, New York: John Wiley \& Sons.

[9] Catlow, F. R. A., Parker, S. C. and Allen, M. P. (1990). Computer Modelling of Fluids, Polymers, and Solids. Series C: Mathematical and Physical Sciences, 293, Boston: Kluwer.

[10] Chen, D. et al. (1995). Hydrodynamic model of temperature change in open ionic channels, Biophysical J., 69, 2304-2322.

[11] Chen, D. et al. (1997). Permeation through the calcium release channel (CRC) of cardiac muscle, Biophys. J., 72, A108.

[12] Chen, D. P., Lear, J. and Eisenberg, R. S. (1997). Permeation through an open channel. Poisson-NernstPlanck theory of a synthetic ionic channel, Biophys. J., 72, 97-116.

[13] Chen, D. P., Nonner, W. and Eisenberg, R. S. (1995). PNP theory fits current-voltage (IV) relations of a neuronal anion channel in 13 solutions, Biophys. J., 68, A370.

[14] Conley, E. C. (1996). The Ion Channel Facts Book. I. Extracellular Ligand-gated Channels, 1, New York, Academic Press.

[15] Conley, E. C. (1996). The Ion Channel Facts Book. II. Intracellular Ligand-gated Channels, 2, New York, Academic Press. 
[16] Conley, E. C. (1997). The Ion Channel Facts Book. III. Inward Rectifier \& Intercellular Channels, 3, New York, Academic Press.

[17] Cowan, S. W. et al. (1992). Crystal structures explain functional properties of two E coli porins, Nature, 358, 727-733.

[18] Davis, M. E. and McCammon, J. A. (1990). Electrostatics in biomolecular structure and dynamics, Chem. Rev., 90, $509-521$.

[19] Eisenberg, R. S. (1996). Computing the field in proteins and channels, J. Membrane Biol., 150, 1-25.

[20] Elber, R. et al. (1993). MOIL: A molecular dynamics program with emphasis on conformational searches and reaction path calculations., in Statistical Mechanics, Protein Structure and Protein-Substrate Interactions, Plenum Press, New York.

[21] Evans, D. J. and Morriss, G. P. (1990). Statistical Mechanics of Nonequilibrium Liquids, New York, Academic Press.

[22] Haile, J. M. (1992). Molecular Dynamics Simulation, New York, John Wiley \& Sons.

[23] Hille, B. (1992). Ionic Channels of Excitable Membranes 2nd ed. Sunderland, Sinauer Associates Inc.

[24] Honig, B. and Nichols, A. (1995). Classical electrostatics in biology and chemistry. Science, 268, 1144-1149.

[25] Hoover, W. G. (1991). Computational Statistical Mechanics, New York, Elsevier.

[26] Jeanteur, D. et al. (1994). Structural and functional alterations of a colicin-resistant mutant of ompF porin from Eschericia coli, Proc. Natl. Acad. Sci. USA, 91, $10675-10679$

[27] Lou, K.-L. et al. (1996). Structural and functional characterization of ompF porin mutants selected for large pore size. I. Crystallographic analysis, J. Biol. Chem., 271, 20669-20675.

[28] Lundstrom, M. (1992). Fundamentals of Carrier Transport, NY, Addison-Wesley.

[29] Mareschal, M. and Holian, B. L. (1992). Microscopic Simulations of Complex Hydrodynamic Phenomena, New York, Plenum Press.

[30] Newman, J. S. (1991). Electrochemical Systems 2nd ed. Englewood Cliffs, NJ, Prentice-Hall.
[31] Peracchia, C. (1994). Handbook of Membrane Channels, New York, Academic Press.

[32] Rudy, B. and Iverson, L. E. (1992). Ion Channels, Methods in Enzymology, 207, New York, Academic Press.

[33] Saint, N. et al. (1996). Structural and functional characterization of ompF porin mutants selected for large pore size. II. Functional characterization, J. Biol. Chem., 271, 20676-20680.

[34] Sakmann, B. and Neher, E. (1995). Single Channel Recording, Second ed. New York, Plenum.

[35] Schultz, S. G. et al. (1996). Molecular Biology of Membrane Disorders, New York, Plenum.

[36] Stryer, L. (1995). Biochemistry Fourth ed. New York, W. H. Freeman.

[37] Tang, J. et al. (1997). Permeation through porin and its mutant G119D. Biophysical Journal, 72, A108.

[38] Weiss, M. S. and Schulz, G. E. (1992). Structure of porin refined at $1.8 \AA$ resolution. J. Mol. Bio., 227, 493-509.

\section{Authors' Biography}

Bob Eisenberg was born in Brooklyn, NY, in 1942, and educated at Horace Mann School, then Harvard. He was trained as a biochemist by John Edsall, as a biophysicist by Bernard Katz, as a physiologist by Andrew Huxley, and (from afar) by Alan Hodgkin. Working at UCLA from $1968-$ 1976, he learned some applied mathematics from Julian Cole and Victor Barcilon while studying current flow in cells, chiefly muscle. Moving to Rush Medical College, Chicago, in 1976, he became the Bard Professor and Chairman of their Department of Molecular Biophysics and Physiology. He has been working on self-consistent models of ionic channels for some ten years. 

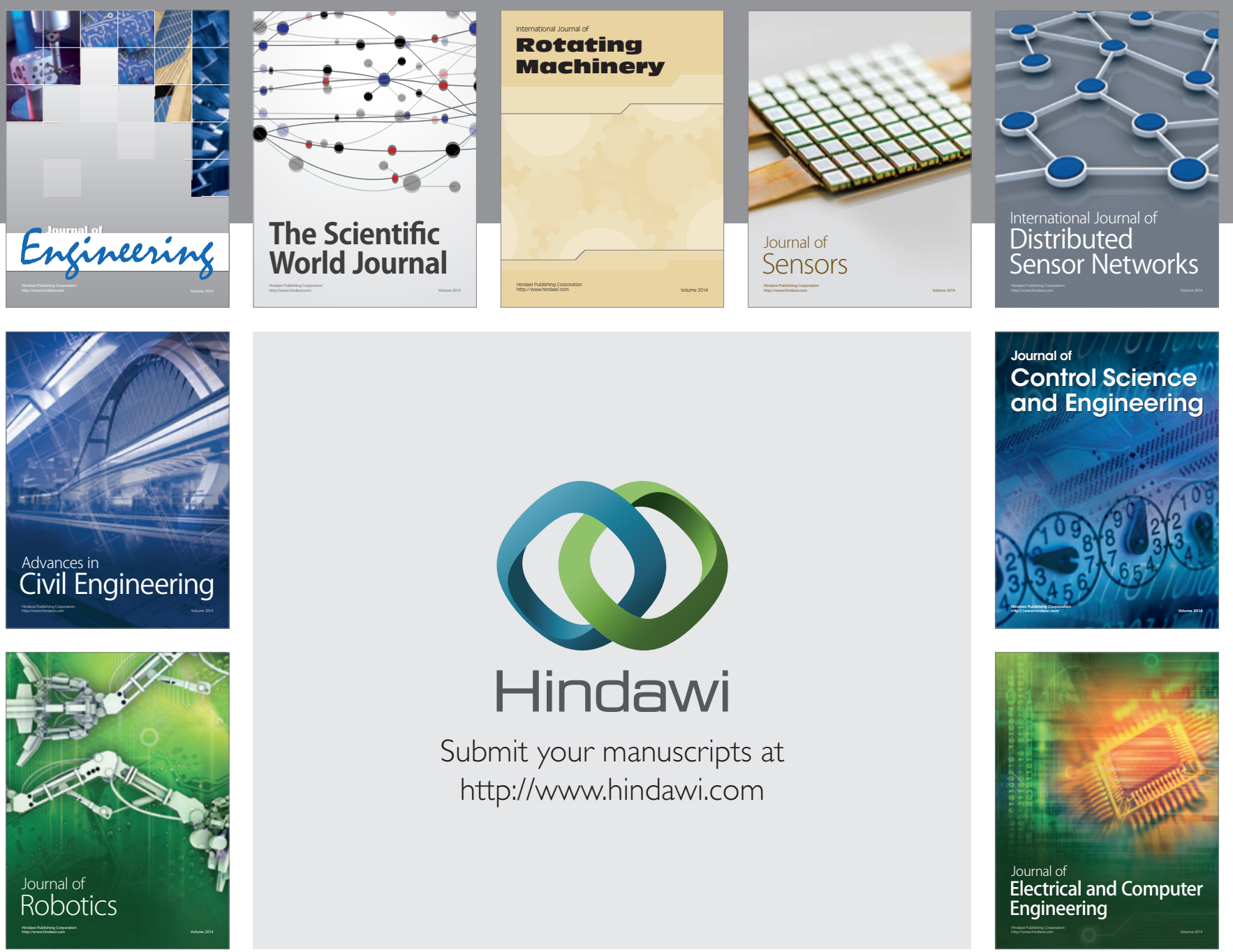

Submit your manuscripts at

http://www.hindawi.com
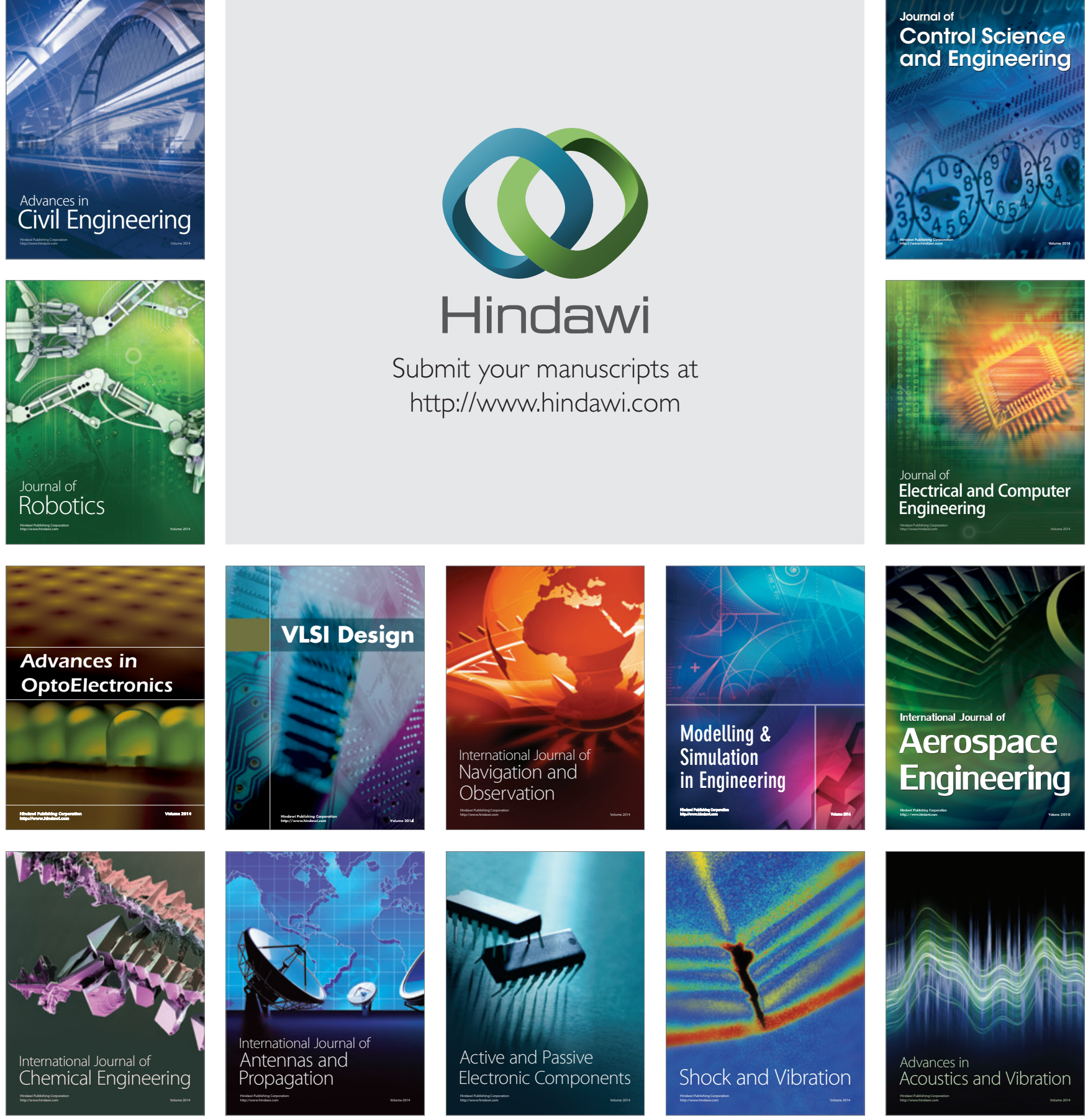